\title{
Editors’ Address
}

\section{Dierk Vorwerk $\cdot$ Ziv J. Haskal}

Published online: 12 December 2008

(C) Springer Science+Business Media, LLC 2008

Dear Readers,

The year 2009 is a special one for Cardiovascular and Interventional Radiology (CVIR). Our publisher, Springer Science+Business Media, LLC, and the Cardiovascular and Interventional Radiological Society of Europe (CIRSE) have agreed to share ownership of CVIR from 2009 onward. This accord significantly strengthens the bonds between CVIR, the official journal of CIRSE, and the European community of interventional radiologists. To celebrate this milestone, we introduce the new cover of CVIR. We hope that this eye-catching and modern look will find favour with you as it does with us. It also brings CVIR into the more unified appearance of other CIRSE publications.

With this visual update come other important CVIR changes. In the past year, CIRSE established joint membership affiliations with Indian, Chinese, and most of the European national societies of interventional radiology. To mirror these developments, we have introduced changes in CVIR's editorial structure. The Editors are pleased to welcome Drs. Sanjiv Sharma as Associate Editor (India) and Robert Morgan as Associate Editor (Review Articles).

Finally, we announce the completion of Dr. Yasuaki Arai's term as the Japanese Society of Interventional Radiology (JSAIR) Editor of CVIR. Dr. Arai has provided a strong editorial voice to $C V I R$, which is made manifest in the many important contributions made to CVIR from Japan. We thank Professor Arai for his years of great service and dedication to the journal. It is our pleasure to announce and welcome the new JSAIR editor in our group, Professor Shozo Hirota of the Hyogo College of Medicine. 